\title{
Merger rates for early-type galaxies: combining clustering and luminosity function measurements
}

\author{
Nelson D. Padilla ${ }^{1}$ Eric Gawiser ${ }^{2}$ Daniel Christlein ${ }^{3}$ \\ and Danilo Marchesini ${ }^{3}$ \\ ${ }^{1}$ Universidad Católica de Chile, Chile, email: npadilla@astro.puc.cl ${ }^{2}$ Rutgers University, \\ USA $;{ }^{3}$ Tufts University, USA; ${ }^{4}$ Max Planck Institute for Astrophysics, Garching, Germany.
}

\begin{abstract}
We present a study of the evolution of early-type galaxies (ETGs) that combines luminosity function and clustering measurements. This technique shows that ETGs at a given redshift evolve into brighter galaxies in the rest-frame passively evolved optical at lower redshifts. Notice that this indicates that a stellar-mass selection at different redshifts does not necessarily provide samples of galaxies in a progenitor-descendant relationship. The comparison between high redshift ETGs and their likely descendants at $z=0$ points to a higher number density for the progenitors by a factor 3 to 11, implying the need for mergers to decrease their number density by today. Because the progenitor-to-descendant ratios of luminosity density are consistent with the unit value, our results show no need for strong star-formation episodes in ETGs since $z=1$, which indicates that the needed mergers are dry, i.e. gas free.
\end{abstract}

Keywords. galaxies: evolution, interactions, luminosity function, mass function

\section{Methods and results}

We present the analysis of the evolution of MUSYC (MUlti-wavelength Survey by Yale- Chile, Gawiser et al. 2006) early type galaxies (ETG) by Padilla et al. (2011, P11). This analysis uses luminosity and correlation functions for MUSYC ETG galaxies with rest-frame $M_{R}(0)<19.7$ corrected by passive evolution, which corresponds to $M_{\text {stellar }}>10^{10} M_{\odot} / h$. The former are extracted from Christlein et al. (2009), and the latter from Padilla et al. (2010).

The method consists of selecting ETGs at a given redshift to then use their measured correlation function to obtain the dark-matter (DM) halo mass of their hosts. DM haloes of this mass are followed to $z=0$ in a numerical simulation. At $z=0$, their average DM mass or, equivalently, clustering amplitude are measured. We then find observational samples of $z=0$ ETGs with the same clustering amplitude, and define these as the descendants of the high redshift MUSYC ETG. Using the luminosity function of the high and low redshift samples, we measure their number and luminosity densities. The former are lower in the descendant samples which indicates the need for mergers, whereas the latter show no change with redshift, indicating no need for star formation, which suggests the mergers would be dry.

\section{References}

Christlein D., Gawiser E., Marchesini D., \& Padilla N., 2009, MNRAS, 400, 429

Gawiser E., et al. 2006, ApJS, 162, 1

Padilla N., Christlein D., Gawiser E., \& Marchesini D., 2011, A\&SA, 531, 142

Padilla N., Christlein D., Gawiser E., Gonzalez R., Guaita L., \& Infante L., 2010, MNRAS, 409, 184 